\title{
Artigo/Article
}

\section{Fatores relacionados à ocorrência de leishmaniose tegumentar no Vale do Ribeira}

\author{
Factors relating to occurrences of cutaneous leishmaniasis in the Ribeira Valley
}

\author{
Alessandra Ferreira da Silva ${ }^{1}$, Maria do Rosário Dias de Oliveira Latorre ${ }^{1}$ e Eunice Aparecida Bianchi Galati ${ }^{1}$
}

\begin{abstract}
RESUMO
Introdução: Este estudo teve como objetivo identificar fatores ambientais e sociais determinantes na incidência da leishmaniose tegumentar americana no Vale do Ribeira no período de 1998 a 2006. Métodos: Foram utilizados dados secundários de domínio público dos 23 municípios que integram a região. $\mathrm{O}$ intervalo de tempo foi dividido em três períodos, pelas características gráficas dos coeficientes de incidência, os quais foram submetidos à análise por regressão linear múltipla. Resultados: Para o período de 1998 a 2000, as variáveis correlacionadas com a LTA foram índice de desenvolvimento humano médio $(\mathrm{p}=0,007)$, renda per capita $(\mathrm{p}=0,390)$ e grau de urbanização $(\mathrm{p}=0,079)$. No período de 2001 a 2003 e 2004 a 2006 as variáveis correlacionadas com LTA foram: a existência de flebotomíneos $(\mathrm{p}=0,000$ e $\mathrm{p}=0,001)$ e a população urbana média $(\mathrm{p}=0,007$ e $\mathrm{p}=0,001)$. Conclusões: Esses dados demonstram a tendência de pauperização e urbanização da doença.
\end{abstract}

Palavras-chaves: Leishmaniose tegumentar americana. Vale do Ribeira. Regressão linear múltipla. Fatores sociais. Fatores ambientais. Dados secundários.

\begin{abstract}
Introduction: This study aimed to identify the environmental and social factors that determined occurrences of American cutaneous leishmaniasis in the Ribeira valley region of the State of São Paulo between 1998 and 2006. Methods: Secondary public-domain data from the 23 municipalities forming this region were used. The study period was divided into three ranges based on the graphical characteristics of the incidence coefficients. These data were subjected to analysis using multiple linear regression. Results: Over the period 1998 to 2000 , the variables correlated with American cutaneous leishmaniasis were mean human development index $(\mathrm{p}=0.007)$, per capita income $(\mathrm{p}=0.390)$ and degree of urbanization ( $p=0.079$ ). Over the periods 2001 to 2003 and 2004 to 2006, the variables correlated with American cutaneous leishmaniasis were the presence of sandflies $(p=0.000 ; p=0.001)$ and mean urban population $(\mathrm{p}=0.007 ; \mathrm{p}=0.001)$. Conclusions: These data show the trend towards urbanization and impoverishment of the disease.
\end{abstract}

Key-words: American cutaneous leishmaniasis. Ribeira valley. Multiple linear regression. Social factors. Environmental factors. Secondary data.

1. Departamento de Epidemiologia, Faculdade de Saúde Pública, Universidade de São Paulo, São Paulo, SP.

Endereço para correspondência: Dra Alessandra Ferreira da Silva. Depto de Epidemiologia/FSP/USP. Dr. Arnaldo 715, Cerqueira Cesar, 01246-904 São Paulo, SP.

Tel: 5511 3061-7737.

e mail: aleferreira@usp.br

Recebido para publicação em 05/12/2008

Aceito em 05/12/2009

\section{INTRODUÇÃO}

As leishmanioses constituem doenças infectoparasitárias cujos agentes etiológicos são protozoários tripanosomatídeos do gênero Leishmania transmitidos por flebotomíneos (Diptera, Psychodidae). Segundo a Organização Mundial de Saúde (OMS), são consideradas antropozoonoses e integram o conjunto das seis doenças tropicais mais importantes no Velho Mundo e nas Américas ${ }^{1,2,3}$. A forma tegumentar é caracterizada por úlcera cutânea e/ou mucosa, apresenta várias espécies de agentes etiológicos classificados em dois subgêneros Leishmania, que no tubo digestivo do vetor apresenta comportamento suprapilórico e Viannia, com comportamento peripilórico e com várias espécies de ocorrência no Brasil. O subgênero Leishmania (Leishmania) amazonensis é espécie deste grupo com a mais ampla distribuição, tendo sido notificada em todas as regiões brasileiras ${ }^{4}$. Os principais reservatórios são pequenos roedores silvestres 5 . Diferentes formas de manifestação clínica dependem da interação agente hospedeiro e da saliva do vetor modulando a resposta deste $^{6}$. Assim, na dependência desta interação, a dinâmica da leishmaniose tegumentar pode apresentar características clínicas e epidemiológicas distintas, entre os locais de ocorrência em função das variáveis relacionadas aos parasitos, aos vetores, aos ecossistemas e aos processos sociais de produção e de uso do solo ${ }^{1-4,7-15}$.

A leishmaniose tegumentar americana (LTA) tem apresentado um aumento do número de casos e ampliação de sua ocorrência geográfica nos últimos 20 anos. É encontrada, atualmente, em todos os estados brasileiros, sob diferentes perfis epidemiológicos e padrões de transmissão em decorrência das modificações sócioambientais ${ }^{3,7,9,18}$. Variações cíclicas na incidência da leishmaniose seriam influenciadas fortemente por fatores geográficos e climáticos, responsáveis pelas flutuações nas populações de flebotomíneos ${ }^{3,9}$. 
No Estado de São Paulo, a LTA encontra-se em expansão e a região do Vale do Ribeira apresenta ao longo do período, as taxas de incidência mais altas ${ }^{4,16,17}$. A influência da cobertura vegetal, relevo e presença de flebotomíneos, por espécies, foram correlacionados com a incidência de LTA no Estado de São Paulo ${ }^{18}$. O presente estudo teve como objetivo identificar fatores ambientais e sociais determinantes na incidência da LTA no Vale do Ribeira no período de 1998 a 2006.

\section{MÉTODOS}

Este é um estudo ecológico, ou de agregado, realizado com dados referentes ao Vale do Ribeira que é constituído por uma faixa de terra de 2.830.666 hectares $\left(28.306 \mathrm{~km}^{2}\right)$ que abrange os Estados de São Paulo (1.711.533 hectares) e do Paraná (1.119.133 hectares).É assim denominado por compreender a Bacia Hidrográfica do Rio Ribeira de Iguape e o Complexo Estuário Lagunar de Iguape, Cananéia e Paranaguá. Estão compreendidos no Vale do Ribeira, 39 municípios, sendo que, 23 e 16 deles, pertencem respectivamente ao Estado de São Paulo e Paraná ${ }^{18,20,21}$ (Figura 1).

Foram selecionados como unidade de estudo os municípios pertencentes ao Vale do Ribeira de Iguape paulista (23 municípios) os quais pertencem às regiões administrativas de Registro, Sorocaba e Grande São Paulo ${ }^{19}$, listados a seguir: Apiaí, Barra do Chapéu, Barra do Turvo, Cajati, Cananéia, Eldorado, Iguape, Ilha Comprida, Iporanga, Itaóca, Itapirapuã Paulista, Itariri, Jacupiranga, Juquiá, Juquitiba, Miracatu, Pariquera-Açu, Pedro de Toledo, Registro, Ribeira, São Lourenço da Serra, Sete Barras e Tapiraí. Esses municípios foram analisados por suas características ambientais e sociais e pela suas taxas de incidência de LTA (Figura 1).
O período estudado compreende os anos de 1998 a 2006, agrupados em três intervalos de três anos. O primeiro intervalo é composto pelos anos de 1998 a 2000, do qual foi obtido coeficiente de incidência médio (coefm1tr), o segundo, pelos anos de 2001 a 2003 (coefm2tr) e o terceiro pelos anos de 2004 a 2006 (coefm3tr). Esta divisão baseou-se em distintas curvas obtidas na representação gráfica de taxas de incidência e/ou do número de casos.

As principais bases de dados secundários utilizadas neste estudo foram: Secretaria Estadual de Saúde, Sistema de Informação de Agravos de Notificação do Centro de Vigilância Epidemiológica (SINAN - CVE), de onde se obteve dados relativos ao número de casos e a distribuição geográfica dos casos. Superintendência de Controle de Endemias (SUCEN), com informações sobre a presença dos flebotomíneos e sua ocorrência geográfica. A Fundação Sistema Estadual de Análises de Dados (SEADE) e o Instituto Brasileiro de Geografia e Estatística (IBGE), que forneceram dados sobre população humana e condições demográficas. Centro Integrado de Informações Agrometeorológicas (CIIAGRO) do Instituto Agronômico (IAC), o Centro de Pesquisas Meteorológicas e Climáticas Aplicadas a Agricultura (CEPAGRI) da Universidade de Campina (UNICAMP) e da Empresa Brasileira de Pesquisa Agropecuária (EMBRAPA) com dados sobre condições climáticas da região. As bases de dados da Companhia de Recursos Minerais (CPRM), do Serviço Geológico do Brasil, do Comitê da Bacia Hidrográfica do Ribeira de Iguape e Litoral Sul (CBH-RB) e da Secretaria de Estado do Meio Ambiente, Instituto Florestal também do Instituto Geográfico e Cartográfico (IGC) forneceram dados sobre condições geográficas.

O teste não paramétrico Kolmogorov Smirnov foi utilizado para testar a normalidade das variáveis. A análise estatística foi feita considerando os coeficientes de incidência dos três períodos como

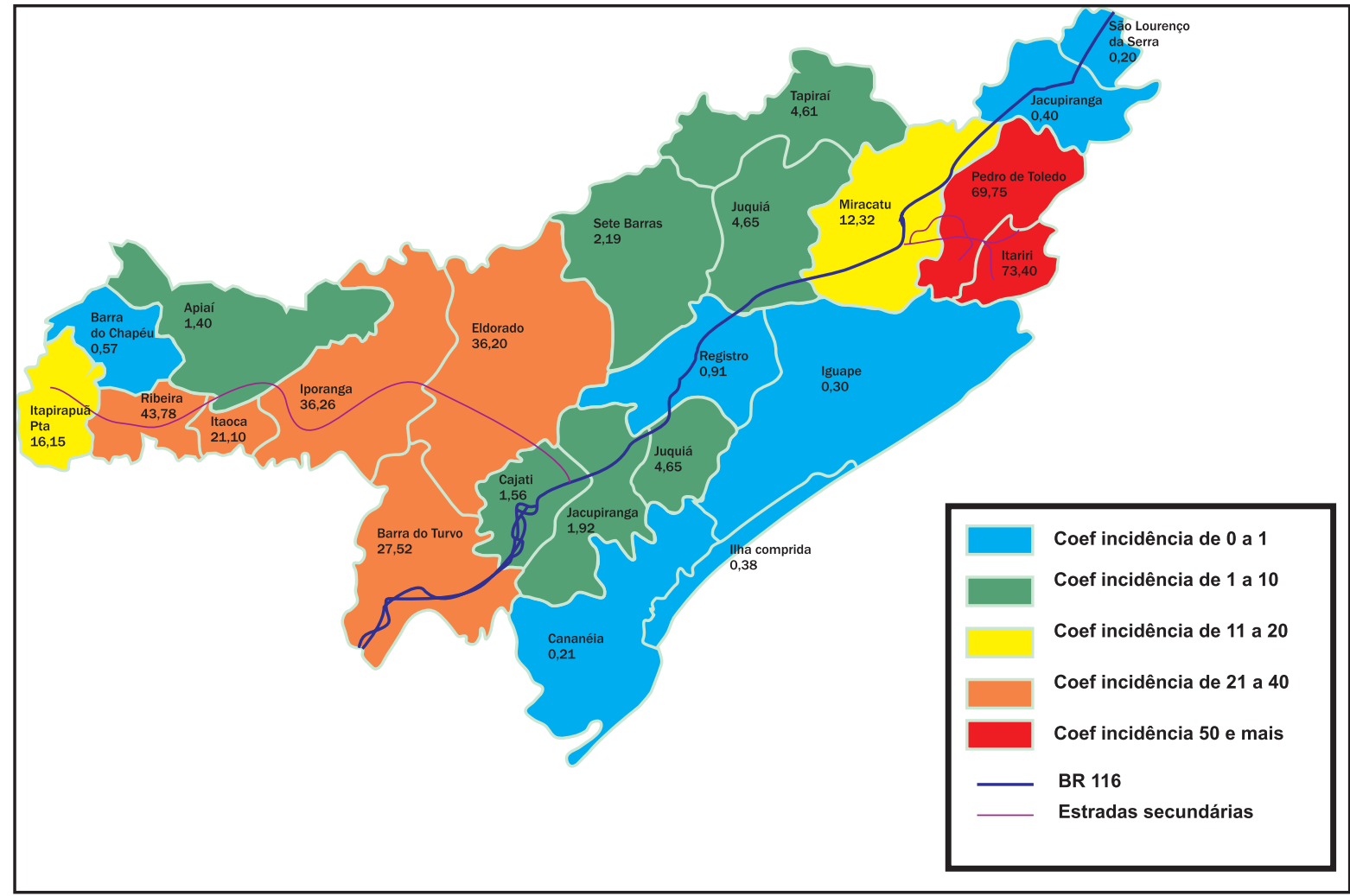

FIGURA 1 - Municípios do Vale do Ribeiro de Iguape segundo taxa de incidência média de leishmaniose tegumentar americana no período de 1998 a 2006. 
variáveis dependentes. Nesta análise, a variável dependente foi transformada em logaritmo na base 10. As variáveis independentes utilizadas neste estudo foram: de natureza sociodemográfica (população média, população rural média, população urbana média, taxa de analfabetismo para maiores de 15 anos, índice de desenvolvimento humano, crescimento geométrico populacional de 1991 a 2000, grau de crescimento geométrico populacional de 2000 a 2007, densidade demográfica, pessoas com mais de 25 anos de idade e menos de oito anos de estudo e renda per capita), relacionadas às condições climáticas (temperatura do ar média, temperatura do ar máxima, temperatura do ar mínima, chuva acumulada máxima e chuva acumulada por ano) e de natureza político-geográficas efísico-biológica (total deáreas de conservação federal, total de área de conservação estadual, existência de parque temático emárea rural, existência de cortiços, número de distritos industriais em área rural, domicílios rurais, existência de favelas, grau de urbanização, domicílios urbanos, presença de indústrias em área rural, domicílios com espaço suficiente, altitude e existência de flebotomíneos).

A análise estatística foi feita primeiramente calculando-se o coeficiente de correlação de Spearman entre os coeficientes de incidência de LTA e as variáveis independentes quantitativas. Também foi feito o teste de comparação de médias dos coeficientes de incidência de LTA segundo as variáveis independentes quantitativas, utilizando o teste de Mann-Whitney. Foram selecionadas todas as variáveis com $\mathrm{p}<0,200$ nestas análises para o processo de modelagem múltipla. Foram feitos três modelos de regressão linear múltipla, utilizando o processo stepwise forward selection.

A variável independente permaneceu no modelo múltiplo se fosse estatisticamente significativa $(p<0,05)$ e/ou fosse variável de controle. Foram avalizados a homocedasticidade e os possíveis vieses do modelo através da análise de resíduos e todas as suposições foram observadas. A análise dos dados foi feita no programa SPSS para Windows versão 15.0 .

\section{Ética}

Segundo o Comitê de Ética em Pesquisa da FSP, a pesquisa embora envolva dados relativos a seres humanos, estes são procedentes de banco de dados de uso e acesso público. Sendo assim, não há necessidade de ser submetido à aprovação.

\section{RESULTADOS}

A Tabela 1 apresenta os coeficientes de correlação entre LTA e as variáveis independentes quantitativas. As variáveis que mais se correlacionarem com a LTA foram as sócio-demográficas, em especial no período de 1998 a 2000. Neste período, também teve correlação positiva, o número de domicílios urbanos. No período de 2001 a 2003, nenhuma correlação foi estatisticamente significativa. No último período, o coeficiente de incidência de LTA teve correlação positiva com o crescimento geométrico populacional de 2000 a 2007, com a população urbana média, com o número de domicílios urbanos e com o grau de urbanização médio.

$\mathrm{O}$ primeiro período demonstra ser uma fase endêmica. $\mathrm{O}$ segundo e terceiro períodos são fases epidêmicas da doença, com aumento da incidência, porém no segundo período existem picos bastante acentuados (Figura 2).

Comparando as médias (Tabela 2), verifica-se que no período de 1998 a 2000, todas as médias foram semelhantes. Já nos períodos 2001 a 2003 e 2004 a 2006, os coeficientes de incidência de LTA foram estatisticamente maiores nos municípios em que havia registros de flebotomíneos (respectivamente, 0,20 x 33,58; $\mathrm{p}<0,001$

TABELA 1 - Coeficientes de correlação de Spearman entre coeficiente médio de leishmaniose tegumentar americana e condições sociodemográficas, climáticas e geográficas, no Vale do Ribeira, 1998-2006.

\begin{tabular}{|c|c|c|c|c|c|c|}
\hline \multirow[b]{2}{*}{ Variáveis } & \multicolumn{2}{|c|}{$1998-2000$} & \multicolumn{2}{|c|}{$2001-2003$} & \multicolumn{2}{|c|}{$2004-2006$} \\
\hline & $\mathbf{r}$ & $\mathbf{p}$ & $\mathbf{r}$ & $\mathbf{p}$ & $\mathbf{r}$ & $\mathbf{p}$ \\
\hline Crescimento geométrico populacional de 2000 a 2007 (\% a.a) & 0,40 & 0,059 & 0,22 & 0,307 & 0,42 & 0,046 \\
\hline Crescimento geométrico populacional de 1991 a 2000 (\% a.a) & 0,42 & 0,048 & 0,21 & 0,331 & 0,40 & 0,062 \\
\hline Densidade demografica $\left(\mathrm{hab} / \mathrm{km}^{2}\right)$ & 0,34 & 0,107 & 0,11 & 0,627 & 0,25 & 0,254 \\
\hline Índice de desenvolvimento humano médio & 0,49 & 0,020 & 0,16 & 0,463 & 0,37 & 0,080 \\
\hline Pessoas com + de 25 anos e - de 8 anos de estudo(\%) & 0,25 & 0,240 & 0,06 & 0,783 & 0,23 & 0,295 \\
\hline População média & 0,43 & 0,041 & 0,26 & 0,230 & 0,40 & 0,059 \\
\hline População rural média & 0,36 & 0,088 & 0,12 & 0,590 & 0,23 & 0,284 \\
\hline Populacão urbana média & 0,43 & 0,042 & 0,31 & 0,153 & 0,45 & 0,033 \\
\hline Renda per capita (salários mínimos) & 0,41 & $\mathbf{0 , 0 5 0}$ & 0,62 & 0,780 & 0,29 & 0,176 \\
\hline Taxa de analfabetismo em pessoas +15 anos (\%) & 0,53 & 0,009 & 0,15 & 0,480 & 0,36 & 0,087 \\
\hline Chuva acumulada por ano (mm) & 0,15 & 0,483 & 0,17 & 0,426 & 0,25 & 0,250 \\
\hline Chuva acumulada máxima por ano $(\mathrm{mm})$ & 0,24 & 0,277 & 0,20 & 0,360 & 0,31 & 0,150 \\
\hline Temperatura do ar máxima $\left({ }^{\circ} \mathrm{C}\right)$ & 0,33 & 0,128 & 0,33 & 0,129 & 0,36 & 0,095 \\
\hline Temperatura do ar média $\left({ }^{\circ} \mathrm{C}\right)$ & 0,31 & 0,152 & 0,33 & 0,122 & 0,35 & 0,102 \\
\hline Temperatura do ar mínima $\left({ }^{\circ} \mathrm{C}\right)$ & 0,18 & 0,418 & 0,19 & 0,385 & 0,19 & 0,387 \\
\hline Altitude (m) & 0,26 & 0,225 & 0,25 & 0,233 & 0,30 & 0,168 \\
\hline Domicílios com espaço suficiente em (\%) (no) & 0,19 & 0,389 & 0,10 & 0,640 & 0,16 & 0,471 \\
\hline Domicílios rurais $\left(\mathrm{n}^{\mathrm{o}}\right.$ ) & 0,36 & 0,090 & 0,11 & 0,612 & 0,23 & 0,283 \\
\hline Domicílios urbanos $\left(\mathrm{n}^{\circ}\right)$ & 0,42 & 0,049 & 0,30 & 0,168 & 0,43 & 0,039 \\
\hline Grau de urbanização médio (\%) & 0,34 & 0,118 & 0,28 & 0,183 & 0,46 & 0,028 \\
\hline
\end{tabular}

r: coeficiente de correlação de Pearson

p: nível de significância 
TABELA 2 - Comparação das médias do coeficiente médio de incidência de leishmaniose tegumentar americana segundo variáveis qualitativas de interesse e período de 1998 a 2006.

\begin{tabular}{|c|c|c|c|c|c|c|c|c|c|c|}
\hline \multirow[b]{2}{*}{ Variável } & \multirow[b]{2}{*}{ Categoria } & \multicolumn{3}{|c|}{1998 a 2000} & \multicolumn{3}{|c|}{2001 a 2003} & \multicolumn{3}{|c|}{2004 a 2006} \\
\hline & & média & dp & $\mathbf{p}$ & média & dp & $\mathbf{p}$ & média & dp & $\mathbf{p}$ \\
\hline \multirow[t]{2}{*}{ Existência de loteamento aprovado na área rural } & não & 12,00 & 17,00 & 0,892 & 27,94 & 48,07 & 0,892 & 14,46 & 17,39 & 0,335 \\
\hline & $\operatorname{sim}$ & 4,23 & 5,09 & & 22,87 & 31,02 & & 9,59 & 14,54 & \\
\hline \multirow[t]{2}{*}{ Total de unidades de conservacão ambiental estadual } & $1-2$ & 7,99 & 16,06 & 0,833 & 16,64 & 36,52 & 0,190 & 8,17 & 12,11 & 0,288 \\
\hline & $3-5$ & 10,13 & 14,36 & & 32,43 & 46,88 & & 16,64 & 18,16 & \\
\hline \multirow[t]{2}{*}{ Existência de parque temático na área rural } & não & 9,55 & 14,97 & 0,589 & 27,27 & 46,34 & 0,947 & 13,23 & 16,75 & 0,853 \\
\hline & $\operatorname{sim}$ & 18,43 & 25,59 & & 23,90 & 33,17 & & 15,78 & 21,53 & \\
\hline \multirow[t]{2}{*}{ Existência de unidade de conservação federal } & não & 10,97 & 16,37 & 0,638 & 21,50 & 33,22 & 0,538 & 12,73 & 14,31 & 0,403 \\
\hline & $\operatorname{sim}$ & 2,42 & 3,12 & & 37,04 & 69,35 & & 12,11 & 22,49 & \\
\hline \multirow[t]{2}{*}{ Existência de conselho municipal de meio ambiente } & não & 8,47 & 13,47 & 0,817 & 16,46 & 23,42 & 0,817 & 11,08 & 13,52 & 0,699 \\
\hline & $\operatorname{sim}$ & 13,15 & 20,61 & & 28,18 & 44,85 & & 12,78 & 15,79 & \\
\hline \multirow[t]{2}{*}{ Presença de distritos industriais no município } & não & 13,71 & 17,57 & 0,255 & 35,15 & 49,19 & 0,400 & 17,19 & 17,45 & 0,094 \\
\hline & $\operatorname{sim}$ & 2,42 & 4,12 & & 11,22 & 24,31 & & 6,39 & 11,19 & \\
\hline \multirow[t]{2}{*}{ Existência de flebotomíneos } & não & 0,49 & 0,62 & 0,117 & 0,20 & 0,49 & $<0,001$ & 0,83 & 0,72 & 0,002 \\
\hline & $\operatorname{sim}$ & 12,15 & 16,34 & & 33,58 & 46,10 & & 16,75 & 16,54 & \\
\hline \multirow[t]{2}{*}{ Existência de cortiços } & não & 13,71 & 16,82 & 0,132 & 37,90 & 16,82 & 0,006 & 18,57 & 16,80 & 0,001 \\
\hline & $\operatorname{sim}$ & 0,53 & 0,58 & & 0,39 & 0,45 & & 1,16 & 1,19 & \\
\hline \multirow[t]{2}{*}{ Existência de favelas } & não & 11,06 & 16,02 & 0,733 & 33,07 & 49,18 & 0,340 & 16,26 & 17,23 & 0,132 \\
\hline & $\operatorname{sim}$ & 7,16 & 14,49 & & 12,47 & 19,85 & & 6,92 & 12,04 & \\
\hline
\end{tabular}

dp: desvio padrão

p: nível de significância

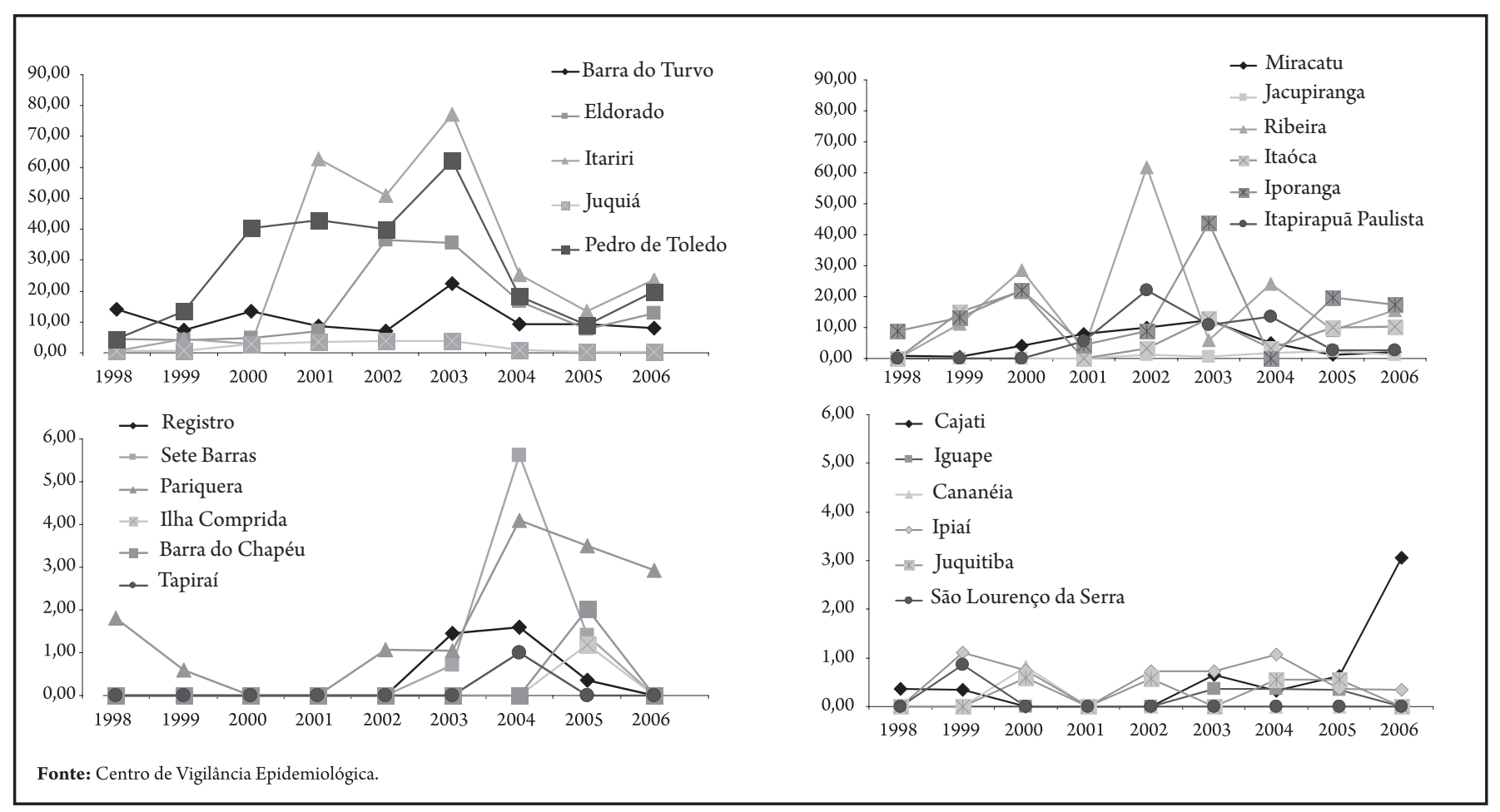

FIGURA 2 - Distribuição de coeficientes de incidência de leishmaniose tegumentar americana no Vale do Ribeira de Iguape, entre 1998 e 2006.

e $0,83 \times 16,75 ; p=0,002)$ e naqueles municípios que tinham cortiços (respectivamente, $37,9 \times 0,39 ; \mathrm{p}=0,006$ e 18,57 x 1,16; $\mathrm{p}=0,001)$.

A Tabela 3 apresenta os resultados dos modelos de regressão finais. Para o período de 1998 a 2000, as variáveis correlacionadas com níveis de incidência da LTA mais elevados foram índice de desenvolvimento humano médio $(\beta=0,001 ; p=0,007)$, renda per capita $(\beta=86,297 ; \mathrm{p}=0,390)$ e grau de urbanização $(\beta=0,962 ; p=0,079)$. Nos períodos de 2001 a 2003 e 2004 a 2006, as variáveis correlacionadas com LTA foram a existência de registros de flebotomíneos $(\beta=29,648$; $p<0,001$ e $\beta=9,036$; $p=0,001)$ e a população urbana média $(\beta=0,989 ; \mathrm{p}=0,007 \mathrm{e} \beta=0,991 ; \mathrm{p}=0,001)$. 
TABELA 3 - Fatores observados à leshmaniose tegumentar americana identificados por meio dos modelos de regressão linear múltipla.

\begin{tabular}{|c|c|c|c|c|c|c|}
\hline \multirow[b]{2}{*}{ Variável } & \multicolumn{2}{|c|}{1998 a 2000} & \multicolumn{2}{|c|}{2001 a 2003} & \multicolumn{2}{|c|}{2004 a 2006} \\
\hline & $\beta$ & $\mathbf{p}$ & $\beta$ & $\mathbf{p}$ & $\beta$ & p \\
\hline Índice de desenvolvimento humano médio & 0,001 & 0,007 & - & - & - & - \\
\hline Renda per capita & 86,297 & 0,390 & - & - & - & - \\
\hline Grau de urbanização & 0,962 & 0,079 & - & - & - & - \\
\hline Existência de flebotomíneos & - & - & 29,648 & 0,000 & 9,036 & 0,001 \\
\hline População urbana média & - & - & 0,989 & 0,007 & 0,991 & 0,001 \\
\hline$r^{2}$ ajustado & 0,710 & & 0,512 & & 0,565 & \\
\hline $\mathrm{p}$ (modelo) & 0,001 & & 0,000 & & 0,000 & \\
\hline
\end{tabular}

$\beta$ : coeficiente de regressão

r2: coeficiente de determinação múltipla

p: nível de significância

\section{DISCUSSÃO}

O Vale do Ribeira embora apresente imenso patrimônio ambiental e cultural, não possui alternativas econômicas adequadas ao desenvolvimento sustentável que permitam a utilização racional desses recursos, segundo entidades não governamentais que atuam na região, como o SOS Ribeira.A presença de extensas áreas com remanescentes de mata atlântica no Vale do Ribeira favorece a presença de flebotomíneos, razão pela qual a maioria dos municípios do Vale do Ribeira apresenta vetores, segundo a SUCEN. Por outro lado, a localização das moradias próximas às matas aumenta as possibilidades de transmissão autóctone de $\mathrm{LTA}^{3}$. O comportamento dos vetores está influenciado por fatores ambientais submetidos à atividade antrópica ${ }^{18}$.

Na investigação sobre causas de diferenças na incidência da LTA, entre os diferentes períodos de tempo, foi possível utilizar modelos múltiplos de regressão com o objetivo de avaliar a extensão, a direção e força da relação (associação). Tratando-se de um estudo ecológico clássico $^{22,23}$.

Modelou-se a LTA (fenômeno), descreveu-se o comportamento da série, estimativas foram feitas e pôde-se tentar avaliar os fatores que influenciaram o comportamento das séries ${ }^{22,23}$.

Este estudo descritivo ecológico usa modelo de regressão buscando explicar a variação na incidência da LTA no Vale do Ribeira, através das variáveis ambientais e sociais. Outros estudos também usaram esse modelo de análise para leishmaniose. Porém, em sua maioria referem-se à leishmaniose visceral, como o trabalho realizado por Oliveira e Araújo ${ }^{24}$, em 2003, investigando a qualidade das ações contra o calazar na Bahia por meio de modelo de regressão; Costa $\mathrm{col}^{25}$, em 1990, realizaram um estudo em Teresina no Piauí, concluiu serem os movimentos migratórios da população em decorrência das secas, o fator responsável pela epidemia ocorrida na região, através de modelo de regressão simples; Moreno $\mathrm{col}^{26}$, em 2002, tiveram como objetivo determinar a prevalência da infecção assintomática por Leishmania chagasi em população urbana exposta ao risco no Piauí em virtude das altas prevalências da leishmaniose visceral canina; e identificar fatores de risco, também usou modelo de regressão logística para análise. Já para leishmaniose tegumentar, há poucos trabalhos com o de $\mathrm{Neves}^{18}$, que encontrou correlações entre fatores ambientais e leishmaniose tegumentar.

Houve a necessidade de três modelos diferentes, e da comparação dos mesmos para verificar se os fatores determinantes que levaram as séries a se comportar de maneira diferenciada seriam os mesmos ou não.
A análise compara três períodos distintos e sequenciais que demonstram coeficientes de incidência de LTA diferentes. No primeiro período de 1998 a 2000, têm-se como variáveis independentes correlacionadas: índice de desenvolvimento humano médio, renda per capita e grau de urbanização. Este período se caracteriza por fase endêmica, na qual a incidência em todo o território é baixa. No segundo período, de 2001 a 2003 nota-se o aparecimento de picos na incidência da doença que não se correlacionaram com flutuações nas chuvas ou na temperatura do ar. Porém, a correlação ocorreu entre o coeficiente de incidência e as variáveis independentes: existência de flebotomíneos e população urbana média. Em 2004 a 2006, observam-se as mesmas variáveis independentes correlacionadas; no entanto, a incidência diminui em alguns municípios e há picos de menor intensidade em outros. O que se pode perceber é uma mudança no padrão do primeiro para os dois outros períodos.

O trabalho aponta para os fatores sociodemográficos como determinantes na incidência de LTA, bem como, a presença do vetor. Segundo $\mathrm{Neves}^{18}$, pode-se supor que a simples detecção de uma das espécies de flebotomíneos de maior importância epidemiológica, já configura fator de risco para a LTA, não sendo este risco potencializado pela detecção de mais de uma espécie.

As variáveis independentes, grau de urbanização, no primeiro período, juntamente a população urbana no segundo e terceiro períodos apontam na direção da urbanização da doença. A leishmaniose tegumentar americana era restrita a trabalhadores que adentravam a mata e considerada doença profissional. Nos anos 50, após intensa destruição florestal a LTA quase desaparece. Aumento significativo da doença ressurge nos anos 80 , com casos autóctones, em novas áreas geográficas e em forma de endemia, na periferia de centros urbanos, acometendo idosos e crianças, classicamente mais restritos ao ambiente doméstico e seus arredores, o que indica a antropização. A espécie vetora mais presente na área é a Nyssomyia intermedia s.lat encontra-se em maior densidade nas áreas endêmicas do estado, já que constitui a espécie predominante no ambiente altamente modificado ${ }^{4,16,18,20}$.

Entretanto, pela diferença entre os tipos de dados e pela necessidade da sua mistura, fato clássico quando são utilizados os estudos de agregados em áreas, não foi possível analisar com profundidade as relações entre clima, relevo e susceptibilidade a erosão. As classificações encontradas desses dados, não permitiram que as variáveis resultantes assumissem distribuições quantitativas, ou mesmo fossem categorizadas a contento para que pudessem ser utilizadas no modelo de regressão. Portanto, a análise quanto a esses 
aspectos poderia ser mais bem investigada, principalmente através de geoprocessamento. Apesar disso, as outras variáveis climáticas testadas não obtiveram correlação com a incidência de LTA no modelo múltiplo final. Dias cols ${ }^{9}$, em 2007, verificaram a interferência de fatores climáticos (temperatura, umidade relativa do ar e pluviosidade) sobre a dinâmica populacional de flebotomíneos e não encontrou uma correlação positiva. Foram levantadas informações sobre legislação e administração pública, que por não apresentarem correlação inicial, não foram testadas, porém esses fatores foram levados em consideração.

Uma das fragilidades dos estudos ecológicos consiste na incapacidade de associar exposição e doença no nível individual, portanto representam níveis de exposição média da população exposta e não exposição individual real. Outra dificuldade é o controle dos efeitos de potenciais fatores de confusão. A qualidade dos dados é variável em virtude de serem provenientes de diferentes fontes. Acresce-se a isto o fato de que pode ocorrer a falta de disponibilidade de informações relevantes, consistindo-se em um dos mais sérios problemas na análise ecológica ${ }^{22,27}$.

Por outro lado, o estudo mostrou que a existência do flebotomíneo e a população susceptível são fatores primordiais para o surgimento da doença e que essa população se torna susceptível, provavelmente, por questões socioeconômicas e demográficas. Apesar de que a existência de flebotomíneos pode ser explicada por uma maior pesquisa entomológica nas áreas aonde já se conhece haver transmissão de LTA, ou mesmo para confirmar se a doença é autóctone. Quanto à população, podemos afirmar que se trata de uma população que cresce, em locais onde a moradia é ruim, em centros urbanos, com baixa renda per capita e baixos índices de desenvolvimento humano e que, além disso, está exposta ao vetor.

\section{CONFLITO DE INTERESSE}

Os autores declaram não haver nenhum tipo de conflito de interesse.

\section{REFERÊNCIAS}

1. Desjeux P. Leishmaniasis: current situation and new perspectives. Comparative Immunology, Microbiology \& Infectious Diseases. Elsevier 2004; 27: 305 318.

2. Guerra JAO, Barbosa MGV, Loureiro ACSP, Coelho CP, Rosa GG, Coelho LIACR. American tegumentary leishmaniasis in children: epidemiological aspects of cases treated in Manaus, Amazonas, Brazil. Cad Saúde Pública 2007; 23: 2215-2223.

3. Secretaria de Vigilância em Saúde. Programa Nacional de DST e AIDS. Recomendações para diagnóstico, tratamento e acompanhamento da co-infecção Leishmania-HIV. Brasília (DF): Ministério da Saúde; 2004.

4. Fundação Nacional de Saúde. Manual de controle da leishmaniose tegumentar americana. Brasília (DF): Ministério da Saúde; 2000.

5. Gontijo B, Carvalho MLR. Leishmaniose tegumentar americana. Rev Soc Bras Med Trop 2003; 36: 71-80.

6. Sacks D, Kamhawi S. Molecular aspects of parasite-vector and vector-host interaction in leishmaniasis. Ann Rev Microbiol 2001; 55: 453-483.

7. Andrade-Filho JD, Galati EAB, Falcão AL. Nyssomyia intermedia (Lutz \& Neiva, 1912) and Nyssomyia neivai (Pinto, 1926) (Diptera: Psychodidae:
Phlebotominae) geographical distribution and epidemiological importance. Mem Inst Oswaldo Cruz 2007; 102: 481-487.

8. Benevenuto Junior P. Geografia e Ecologia da Leishmaniose Tegumentar no Estado do Espírito Santo [Dissertação de Mestrado]. [Rio de Janeiro (RJ)]: Escola Nacional de Saúde Pública Fundação Oswaldo Cruz; 2000.

9. Dias ES, França-Silva JC, Silva JC, Monteiro EM, Paula KM, Gonçalves CM, et al Flebotomíneos (Diptera: Psychodidae) de um foco de leishmaniose tegumentar no Estado de Minas Gerais. Rev Soc Bras Med Trop 2007; 40: 49-52.

10. Galati EAB. Morfologia e Taxonomia. In: Rangel EF, Lainson R (eds) Flebotomíneos do Brasil. Editora FIOCRUZ, Rio de Janeiro; 2003. p. 23-51.

11. Hayashi EE. Estudo da leishmaniose tegumentar americana no Estado do Mato Grosso, no período de 1994 a 1999 [Dissertação de Mestrado]. [Ribeirão Preto (SP)]: Faculdade de Medicina de Ribeirão Preto Universidade de São Paulo; 2004.

12. Marzochi MCA. Leishmanioses no Brasil: as leishmanioses tegumentares. J Bras Med 1992; 63: 82-104

13. Neves VLFC, Aspectos epidemiológicos e avaliação das medidas de controle da leishmaniose visceral americana no Estado de São Paulo, Brasil [Tese de Doutorado]. [São Paulo (SP)]: Faculdade de Saúde Pública Universidade de São Paulo; 2004.

14. Silva DA. Aspectos Ecológicos da fauna flebotomínea (Diptera, psydodidae) e suas implicações na epidemiologia das leishmanioses em Cotia, Estado de São Paulo, Brasil [Dissertação de Mestrado]. [São Paulo (SP)]: Faculdade de Saúde Pública Universidade de São Paulo; 2005.

15. Silva AM, Camargo NJ, Santos SR, Massafera R, Ferreira AC, Postai C, et al Diversidade, distribuição e abundância de flebotomíneos (Diptera: Psychodidae) no Paraná. Neotrop Entomol 2008; 37: 209-225.

16. Domingos MF, Carreri-Bruno GC, Ciaravolo RMC, Galati EAB, Wanderley DMV, Corrêa FMA. Leishmaniose tegumentar americana: flebotomíneos de área de transmissão, no município de Pedro de Toledo, região sul do Estado de São Paulo, Brasil. Rev Soc Bras Med Trop 1998; 31: 425-432.

17. Neves VLFC. Características da transmissão da Leishmaniose Tegumentar Americana no Estado de São Paulo, Brasil [Dissertação de Mestrado]. [São Paulo (SP)]: Faculdade de Saúde Pública Universidade de São Paulo; 1999.

18. Neves VLFC, Gomes AC, Antunes JLF. Correlação da presença de espécies de flebotomíneos (Diptera: Psychodidae) com registros de casos da leishmaniose tegumentar americana no Estado de São Paulo, Brasil. Rev Soc Bras Med Trop 2002; 35: 299-306

19. Comitê da Bacia Hidrográfica do Ribeira de Iguape e Litoral Sul. SIG - RIBEIRA - Relatório de Situação dos Recursos Hídricos da Unidade de Gerenciamento $\mathrm{n}^{\circ} 11 ; 2008$

20. Da-Silva LMR, Cunha PR. A urbanização da leishmaniose tegumentar americana no município de Campinas - São Paulo (SP) e região: magnitude do problema e desafios. An Bras Dermatol 2007; 82: 515-519.

21. Silva-Nunes M, Cavasini CE, Silva NS, Galati EAB. Epidemiology of Cutaneous Leishmaniasis and description of phlebotomine sandfly populations in the city of Acrelandia, Acre, Brazil. Rev Bras Epidemiol 2008; 11: 241-251.

22. Carvalho MS, Santos RS. Análise de dados espaciais em saúde pública: métodos, problemas, perspectivas. Cad Saúde Pública 2005; 21:361-378.

23. Latorre MRDO, Cardoso MRA. Análise de séries temporais em epidemiologia. Rev Bras Epidemiol 2001; 4: 145-152.

24. Oliveira SS, Araújo TM. Avaliação das ações de controle da leishmaniose visceral (calazar) em uma área endêmica do Estado da Bahia, Brasil (1995-2000). Cad Saúde Pública 2003; 19: 1681-1690.

25. Costa CHN, Pereira HF, Araújo MV. Epidemia de leishmaniose visceral no Estado do Piauí, Brasil, 1980-1986. Rev Saúde Publica 1990; 24: 361-372.

26. Moreno EC, Melo MN, Antunes CMF, Lambertucii JR, Serufo JC, Andrade ASR, et al. Epidemiologia da leishmaniose visceral humana assintomática em área urbana, Sabará, Minas Gerais, 1998-1999. Informe Epidemiológico do Sistema Único de Saúde 2002; 11: 37-39.

27. Medronho RA, Carvalho DM, Bloch KV, Luiz RR, Werneck GL. Epidemiologia Editora Atheneu: São Paulo; 2004. 